\title{
A novel classification approach of weld defects based on dual-parameters optimization of PCA and LDA
}

\author{
Weilei Mu ${ }^{1, a}$, Guijie Liu* ${ }^{1, b}$, Peng Liu ${ }^{1, c}$ and Xiaojie Tian ${ }^{1, d}$ \\ ${ }^{1}$ School of Engineer, Ocean University of China, Qingdao 266100, China; \\ atblueapple@126.com, ${ }^{b}$ liuguijie@ouc.edu.cn, cplay5719@126.com, ${ }^{d}$ tianxj20050101@163.com
}

Keywords: defects classification, PCA, LDA.

\begin{abstract}
To improve the classification accuracy of defects, a novel algorithm has been developed based on dual-parameters optimization of the principal component analysis (PCA) and the linear discriminant analysis (LDA). The original defect images are transformed to eigen-defects by PCA which contains all features of these defects. Then, LDA is used to classify eigen-defects. The optimal parameters of PCA and LDA are given when the PCA-LDA model gets the maximum value of classification accuracy. For estimating the actual classification accuracy of the proposed method in a concrete system, Bootstrap evaluation method is introduced. The experimental result demonstrates that the accuracy of this method is $91.12 \%$, which promotes the accuracy by $0.37 \%, 3.61 \%$ and 8.72\% comparing with PCA-SVM, SVM and MLP-ANN.
\end{abstract}

\section{Introduction}

Radiographic testing (RT) is one of the most widely used methods for weld internal defects detection. Thus, many efforts have been devoted to developing an automatic defect classification system, and most methods are based on defect features. Among the various approaches, PCA is one of the most widely used preprocess methods. Vilar proposed a method based on PCA and ANN in which the number of features were reduced by PCA ${ }^{(2)}$. Lim et al selected an optimal subset of features from 25 features set using PCA, and then used the MLP neural network to classify the defect. In classification methods, ANN (artificial neural network) and SVM are two widely used methods. Silva and team proposed a method based on multi-layer ANN to identify the defect type ${ }^{(\underline{3})}$. Aoki and Suga used a three-layer ANN to determine the defect type with ten features input. ${ }^{(4)}$ Wang and Liao extracted 12 characteristics of weld defects, and classified weld defects into different types by multi-layer perceptron (MLP) neural network ${ }^{(5)}$. Shen et al defined 8 defect features and proposed an automatic classification system based on the SVM method ${ }^{(\underline{8}, \underline{9})}$.

In our previous papers, we use the traditional methods to recognition defects in which we extract defect features first, and then preprocess the defect features using PCA (or kernel PCA), classify the defects at last using SVM (support vector machine). The optimal PCA-SVM model performs well in the application, but we couldn't confirm which are the most important features, and which are useless features. Moreover, defects of different classes might have the similar feature values. In this paper, we focus on defect classification with a novel approach as the continuation of our work. We try to transform defect images to eigenspace, and the eigenspace defect (called eigen-defect) contains all features of defect. In this way, it has no trouble in choosing defect features. However, the performance of an automatic defect classification system depends on defect features, and also depends on selected training samples and classification algorithm. To solve the problems mentioned above, we collected 174 defect images from weld radiographic images, and use LDA to transform the eigen-defects to low dimensions space which cluster the defects of same class together.

The rest of this paper can be divided into three parts. In Section 2, we will give a brief introduction of the proposed method and the process procedure of this method. Then, We analyse the relation between parameter value and classification accuracy. At last, we draw a conclusion in Section 4. 


\section{Methodology}

Defect images transformation. The radiograph films are digitized by an X-ray film scanner (JD-RTD) which is developed by our research group and saved as MIM format in 12-bit resolution without compression. Radiograph films which contain five kinds of defects are recommended by inspection experts. Defect images are extracted from the radiographic images. Then, we zoom in (out) the defect image to the standard-size defect image at 60X60 resolution. As we know, the input of PCA and LDA is data in matrix representation, in which each column represents a defect sample. Thus, we need to transform the defect data in matrix representation to a signal in vectorized representation. Assume that ${ }^{I_{i}^{\prime}}$ represents $i$-th defect image in matrix representation, ${ }_{j}(1<j<60)$ represents the $j$-th column of the matrix, the transformed representation is shown as follow:

$$
I_{i}^{\prime}=\left[I_{1} ; \quad \ldots ; \quad I_{j} ; \quad \ldots ; I_{60} ;\right]^{T}
$$

The original defect dataset consists of $n$ defect samples, denoted as $I$ :

$$
I^{\prime}=\left[\begin{array}{lllll}
I_{1}^{\prime}, & \ldots, \quad I_{i}^{\prime}, \quad \ldots, \quad I_{n}^{\prime}
\end{array}\right]
$$

Bootstrap method.Usually, the last step of machine learning model is the classification accuracy estimate. Bootstrap method is usually used to infer on estimates of population parameters when the distribution of population or the statistic is unknown. In defects classification system, the distribution of defects population is unknown because it is impractical to collect the whole concrete defect instances. Thus, we choose bootstrap method for accuracy estimate of defects classification.

In the bootstrap method, the training set denoted as $x_{1}^{*}, x_{2}^{*}, \ldots, x_{n}^{*}$ are randomly selected from the original dataset denoted as $x_{1}, x_{2}, \ldots, x_{n}$, with repositioning. The training set is as large as the original dataset. In this way, there are some data appearing for many times in the training set, while some data do not appear. The remaining of the original dataset which are not picked up is taken as the test set.

In the selection with replacement, the probability of any instance which is not chosen after sampling $n$ times is 0.368, and the calculating expression is shown as Eq.(3).

$(1-1 / n)^{n} \approx 0.368, n \rightarrow+\infty$

Thus, the 0.632 bootstrap evaluation accuracy is defined as Eq.(4).

$$
\delta=\frac{1}{B} \sum_{i=1}^{B}\left(0.632 \delta_{i}+0.368 \delta_{c}\right)
$$

Where $\delta$ means the bootstrap evaluation accuracy, $B$ means the times of bootstrap procedure, $\delta_{i}$ means test accuracy for the $i$-th test set, and $\delta_{c}$ is the optimal test accuracy when the whole dataset is taken as the optimal training set and the optimal test set.

The procedure of classification.The novel approach based on PCA for generating eigendefects data and LDA for classification is proposed, and the process procedure is shown as Figure 1.

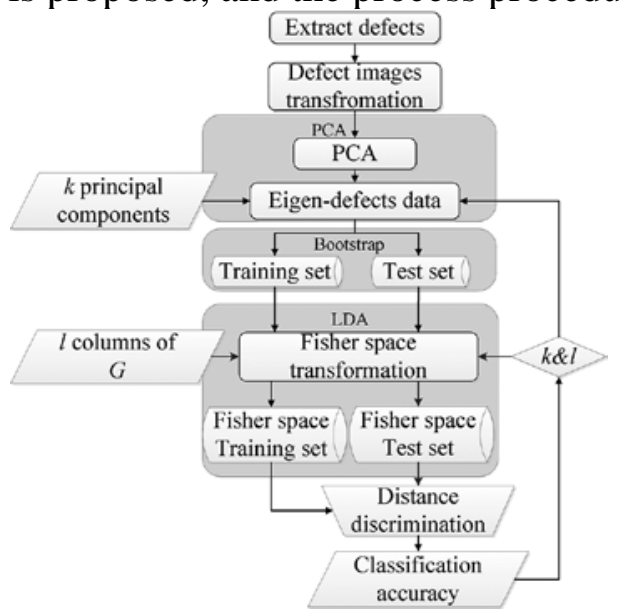

Figure 1. The process procedure

We extract 174 defect instances of five categories, and there are 23 porosities (PO), 29 slag inclusions (SL), 45 lack of penetrations (LP), 25 lack of fusions (LF) and 52 cracks (CR). Then, zoom in (out) the original defect images to the standard-size images of $60 \times 60$ resolution. After that, 
transform each standard-size image to a vector $I_{i}^{\prime} \in I R^{3600 \times 1}(1 \leq i \leq 174)$, and combine the 174 defect vectors to a matrix $I^{\prime} \in I R^{3600 \times 174}$. Use PCA algorithm to transform defcet data in matrix representation to the eigen-defects space data $A \in I R^{3600 \times 174}$. Usually, we choose the first $k$ principal components of the eigen-defects space data as restructured matrix of reduced dimensions, denoted as $A_{k} \in I R^{k \times 174}$.

In this experiment, we make $10<k<145$ and change $k$ by 1 . Because the accumulative contribution rates are $85.20 \%$ and $99.90 \%$, when $k$ are 11 and 144 respectively. The trend line of accumulative contribution rate is shown in Figure 2. Then, we use the Bootstrap method to choose the training set from the eigen-defects matrix $A_{\text {train }} \in I R^{k \times 174}$. The unchosen columns are taken as test set $A_{\text {eest }} \in I R^{k \times x}$. After that, we use LDA algorithm to transform training set and test set to Fisher space. The training set in Fisher space is $A_{\text {train }}^{\prime} \in I R^{\mid \times 174}, A_{\text {train }}^{\prime}=G^{T} A_{\text {train }}$. The test set in Fisher space is $A_{\text {eest }}^{\prime} \in I R^{\mid \times x}, A_{\text {est }}^{\prime}=G^{T} A_{\text {eest }}$. It is easy to execute discrimination in Fisher space because the same class defects are close to each other and defects of different classes are farther away.

In this experiment, we make $1 \leq l \leq k$ and get the classification accuracy under $k$ and $l$. Repeat the steps above 5 times $(B=5)$, we get 5 values of classification accuracy under the same parameters. Then, we obtain the evaluation accuracy of PCA-LDA method under $k$ and $l$. Table 1 shows the test accuracies and the optimal test accuracies in Bootstrap evaluation when $k=19$ and $l=4$.

Table 1. Bootstrap evaluation $(k=19$ and $l=4)$

\begin{tabular}{|c|c|c|c|}
\hline Experiment & $\delta_{i}$ & $\delta_{c}$ & Evaluation accuracy \\
\hline$\# 1$ & 91.43 & 96.55 & 93.31 \\
\hline$\# 2$ & 88.57 & 99.43 & 92.57 \\
\hline$\# 3$ & 82.86 & 96.55 & 87.90 \\
\hline$\# 4$ & 91.43 & 100.00 & 94.58 \\
\hline$\# 5$ & 80.00 & 92.53 & 84.61 \\
\hline \multicolumn{2}{|r|}{ Evaluation accuracy (\%) } & 91.12 \\
\hline
\end{tabular}

Change the value of $k$ and $l$ and repeat the process above, we obtain the values of evaluation accuracy respectively. We use the colour dots to represent the classification accuracy, and red dots mean higher classification accuracy than blue dots, as shown in Figure 3 . When $k=19$ and $l=4$, the PCA-LDA method perform best, and the classification accuracy is $91.12 \%$.

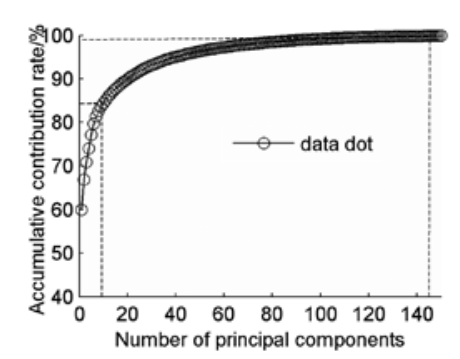

Figure 2. The accumulative contribution rate Figure 3. Classification accuracy of different parameters

\section{Discussion}

As we can see from Figure 3, when the number of principal components increases, the colour of the dots changes from red to blue. It means that the classification accuracy shows a downward trend roughly with the increase of $k$. 

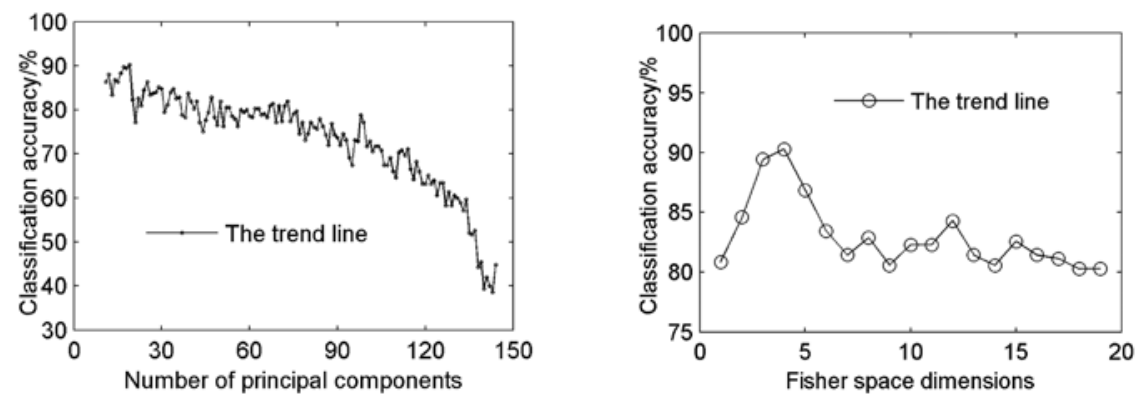

Figure 4. The trend line of classification accuracy Figure 5. The trend line of classification accuracy

We plot a trend line, as $l=4$ and $10<k<145$. More details can be observed in Figure 4 . When the number of principal components is less than 19, defect classification accuracy shows an upward trend. It means that the useful information (signal) for classification increases when the number of principal components increases. When the number is more than 19 , the defect classification accuracy shows a downward trend approximatively. It means that the useless information (noise) for classification begins to decrease.

There are the same phenomena in the relationship between the classification accuracy and Fisher space dimensions, as shown in Figure 5. The trend line shows upward when Fisher space dimensions is less than 4, and approximatively downward when Fisher space dimensions is more than 4.

From the analysis above, we know that there is an optimal quantity of input variables. It means that the optimal input has the maximum signal-to-noise rate and it is beneficial to improve classification accuracy.With the purpose of testing the performance of this method, we compare the PCA-LDA method with PCA-SVM, SVM and multi-layer perceptron artificial neural network (MLP-ANN). We have constructed the optimal PCA-SVM model in our previous work, and the evaluation accuracy of PCA-SVM is $90.75 \%$ as shown in Table 2. Compared with Shen's SVM method and Lim's ANN approach, the proposed PCA-LDA method gets higher classification accuracy.

Table 2. Performance assessment of the methods

\begin{tabular}{|l|l|}
\hline Method & Classification accuracy (\%) \\
\hline PCA-SVM & 90.75 \\
\hline SVM & 87.51 \\
\hline ANN & 82.40 \\
\hline PCA-LDA & 91.12 \\
\hline
\end{tabular}

\section{Conclusion}

In this paper, we propose an effective classification method for defect automatic classification. We use PCA to transform the defect images to eigen-defects, and use LDA to recognize the defect types of eigen-defects. We give the optimal parameters of PCA and LDA, and the optimal PCA-LDA model obtains the maximum classification accuracy. Bootstrap evaluation accuracy of the optimal PCA-LDA model is $91.12 \%$, which is higher than PCA-SVM, SVM and MLP-ANN. Moreover, the proposed method is easy to carry out because it has no trouble in choosing defect features. Briefly speaking, our work makes a little step to improve the defect classification accuracy, and the proposed method is also suitable for classification of other objects, such as faces and fingerprint classification.

\section{Acknowledgment}

This work was supported by the Fundamental Research Funds for the Central Universities (1600-841513003) and the National Natural Science Foundation of China (No. 51175485). Guijie Liu is the corresponding author. 


\section{References}

[1] Y. Wang, Y. Sun, P. Lv and H. Wang, 'Detection of line weld defects based on multiple thresholds and support vector machine', NDT \& E International, Vol 41, No 7, pp 517-524, 2008.

[2] T. Y. Lim, M. M. Ratnam and M. A. Khalid, 'Automatic classification of weld defects using simulated data and an MLP neural network', insight, Vol 49, No 3, pp 154-159, 2007.

[3] R. R. da Silva, L. P. Cala, M. H. S. Siqueira and J. M. A. Rebello, 'Pattern recognition of weld defects detected by radiographic test', NDT \& E International, Vol 37, No 6, pp 461-470, 2004.

[4] K. Aoki and Y. Suga, 'Application of artificial neural network to discrimination of defect type in automatic radiographic testing of welds', ISIJ International(Japan), Vol 39, No 10, pp 1081-1087, 1999.

[5] G. Wang and T. W. Liao, 'Automatic identification of different types of welding defects in radiographic images', NDT \& E International, Vol 35, No 8, pp 519-528, 2002.

[6] V. Vapnik, The nature of statistical learning theory, springer, 1999.

[7] V. Cherkassky and F. M. Mulier, Learning from data: concepts, theory, and methods, Wiley-IEEE Press, 2007.

[8] Q. M. Shen, J. M. Gao and C. Li, 'Automatic classification of weld defects in radiographic images', Insight, Vol 52, No 3, pp 134-139, Mar 2010.

[9] Q. M. Shen and J. M. Gao, 'Improving the classification accuracy of the weld defect by chaos-search-based feature selection', Insight, Vol 52, No 10, pp 530-532, Oct 2010. 